\title{
CORROSIÓN DE ARMADURAS Y SU INSPECCIÓN EN HORMIGONES DE CEMENTO ALUMINOSO
}

\author{
(CORROSION OF REINFORCEMENT AND ITS INSPECTION IN HIGH \\ ALUMINA CEMENT CONCRETE)
}

M. Carmen Andrade, Profesora de Investigación

ICCET/CSIC

ESPAÑA

Fecha de recepción: 13-XI-92

\begin{abstract}
RESUMEN
Hasta fechas recientes no se ha constatado, en toda su magnitud, el problema de durabilidad que supone para el hormigón la corrosión de sus armaduras, de ahí que los problemas iniciales presentados por el cemento aluminoso no se dimensionaran con anterioridad. En la actualidad, el problema que plantea el cemento aluminoso a largo plazo, además de las pérdidas de resistencia mecánica que se han constatado, es la posible corrosión de sus armaduras.

En este trabajo se describen aspectos relativos a los procesos que puede originar la citada corrosión y se dan algunas indicaciones prácticas de cómo evaluarla.
\end{abstract}

SUMMARY

Until recently has not become evident the magnitude of the problem that the corrosion of the reinforcement supposes for concrete technology. That is why, when aluminous cement preliminary degradations appeared it was not foreseen the dimmension of future consequences.. Now, the long term difficulty that aluminous cement presents is, in addition to the loss in mechanical strenght, the possible corrosion of reinforcement.

In present work are described aspects related to the processes which may originate the corrosion and given are some practical indications on how evalute it.

\section{INTRODUCCIÓN}

En el momento presente ya se ha tenido acceso en nuestro país a bibliografía sobre los mecanismos de degradación del cemento aluminoso [1] [2] [3] y las causas que han podido producir los colapsos detectados hasta ahora [4] [5]. Esta divulgación se ha realizado no sólo a nivel de las revistas científicas, sino también a través de las innumerables noticias y artículos de periódicos. Así pues, en el presente trabajo no se van a presentar facetas nuevas que el lector no pueda encontrar en otros trabajos, pero sí se va a intentar una síntesis lo más breve posible dentro de la amplitud del tema.

Se comenzará comentando algunos datos de encuadre histórico para pasar a exponer la situación, desde el punto de vista químico, en que se encuentran las armaduras del hormigón. Después se intentará situar la realidad de la vida útil de estructuras de cemento aluminoso dentro del contexto de la vida útil de las estructuras en general. Se finalizará con algunas recomendaciones en cuanto a la inspección de elementos realizados con cemento aluminoso.

\section{ENCUADRE HISTÓRICO}

Es importante recordar que cuando aparece el hormigón armado, a principios de este siglo, supone una gran esperanza como alternativa a las estructuras de acero que ya por entonces eran profusamente empleadas, pero que presentaban la debilidad de necesitar un mantenimiento continuo debido a los problemas de corrosión. 
El hormigón nace como un material que no necesita mantenimiento y se considera signo de avanzada tecnología e, incluso, arquetipo de belleza, la realización de grandes láminas o terminaciones de hormigón visto sin protección.

Los recubrimientos de la armadura, que entonces se planteaban, no se basaban en requisitos de durabilidad, sino en la necesidad de trasmisión de las propiedades mecánicas (adherencia) entre el hormigón y el acero. Aún hoy los espesores de recubrimiento recogidos en las presentes normativas y códigos se siguen basando en estos criterios con el añadido de la necesaria resistencia contra el fuego, pero en ningún caso se diseñan a partir de los ahora conocidos cálculos sobre tiempos de penetración de los agresivos ambientales. Los problemas de falta de durabilidad no se asocian más que a casos muy particulares de ambientes especialmente agresivos.

En el contexto de considerar al hormigón como un material casi eterno, las carencias que presenta el cemento portland de resistencia química, por ejemplo en ambientes conteniendo sulfatos, hace que se acoja tan favorablemente la aparición de un cemento químicamente mucho más estable frente a estos ataques como se presentó ser el aluminoso en sus primeros años.

Todo este bagaje de "material sin mantenimiento y químicamente estable" con respecto al hormigón hace crisis sólo recientemente, a pesar de que muchos países ya experimentaron graves problemas en hormigones y concretamente prohibieron, alrededor de la década de los 70 , el uso del cemento aluminoso. Es decir, los problemas detectados en el cemento aluminoso no alteraron la confianza general hacia el hormigón armado como material, al achacarse entonces el fenómeno solamente a la conversión de los aluminatos hexagonales en cúbicos, o bien, en el caso particular del cemento alemán, a la presencia de sulfuros en ese cemento concreto.

Es sólo a partir de finales de la década de los 70 y principios de los 80 cuando empiezan a crecer de forma exponencial las necesidades de reparación de estructuras de hormigón de cemento portland, principalmente debidas a la corrosión de las armaduras generada por el uso masivo de sales de deshielo en climas fríos o en ambientes marinos.

La otra causa de corrosión de las armaduras, la carbonatación (reacción del dióxido de carbono del aire con la pasta de cemento de naturaleza alcalina) del recubrimiento, no atrae la atención de los investigadores al principio más que como un fenómeno curioso que, incluso durante algún tiempo, se preconizó como sistema de protección superficial al haberse constatado una mayor densificación de la pasta [6]. De hecho, en relación con la corrosión de las armaduras son muy pocos los trabajos [7] hasta la aparición de los de Venuat en 1976 [8] en el CERILH de París. Los primeros datos cuantitativos de velocidades de corrosión no aparecen publicados hasta 1980 [9] debidos a González, Algaba y Andrade.

En cambio, datos constatando que se produce corrosión de armaduras en hormigones de aluminoso son publicados por diversos autores [10] [11], destacando en nuestro país del Olmo [12], en 1975, y Vázquez, Triviño y Gauna [13], en 1976. En 1978 se publica también, en nuestro país, los primeros datos de velocidad de corrosión en hormigones de aluminoso debidos a Pérez, Triviño y Andrade [14].

Con todo ello, lo que se pretende hacer ver es que aunque en las décadas de los 50 y los 60 se conocían ya los problemas que se podían generar con el uso del cemento aluminoso, no existía conciencia colectiva de que las estructuras de hormigón pudieran ser menos durables que las de acero o las de madera, y mucha menos idea se tenía de una posible corrosión de las armaduras.

En la actualidad las evidencias son aplastantes y la crisis de la necesidad de someter al hormigón a una protección adicional o a un mantenimiento todavía no se ha resuelto satisfactoriamente. Son hechos verificados en estructuras que ya van teniendo 80-100 años de antigüedad, o las que simplemente tienen más de 30 y fueron construidas después de la segunda guerra mundial, que muchas de ellas tienen sus recubrimientos o contaminados con cloruros o, al menos, carbonatados y, por tanto, necesitan pequeñas o grandes reparaciones de muy elevado costo y dudosa eficacia ante el desconocimiento de la evolución futura de los materiales utilizados en la reparación en esos ambientes.

Dado que el fenómeno que más afecta al cemento aluminoso es la carbonatación, a continuación se harán sólo ligeras referencias a los cloruros como agente agresivo para las armaduras, y se va a centrar el argumento principalmente en la carbonatación. 


\section{CARBONATACIÓN Y CORROSIÓN DE ARMADURAS}

Cuando se hidrata un cemento portland se genera hidróxido cálcico que aporta una elevada alcalinidad $(\mathrm{pH}$ entre 12,6 y 14) a la fase acuosa que queda aprisionada en los poros del hormigón. El exceso de hidróxido cálcico que se genera precipita en estos poros y constituye lo que se llama "la reserva alcalina", ya que se puede ir redisolviendo cuando el $\mathrm{pH}$ de la fase acuosa tienda a neutralizarse. Esta reserva alcalina actúa por lo tanto como un tampón químico.

De esta manera el acero en contacto con cemento portland hidratado aporta una protección de tipo químico basada en el elevado $\mathrm{pH}$ de la fase acuosa. El recubrimiento de hormigón se considera una barrera física entre la armadura y el medio ambiente (Fig. 1).

Si este recubrimiento mantiene sus características químicas y físicas, esta protección se mantiene indefinidamente.

Sin embargo, existen dos factores medioambientales que pueden alterar esta estabilidad: la presencia de dióxido de carbono en el aire y la de cloruros en ambientes marinos. Además está la posibilidad de contacto con ambientes especialmente agresivos como son los industriales.

El dióxido de carbono, al ser una sustancia ácida, cuando se disuelve en agua reacciona con las sustancias alcalinas de la pasta de cemento y provoca una neutralización química conocida como "carbonatación". Da lugar a una despasivación generalizada de la armadura (Fig. 2).

El caso de los cloruros es diferente, ya que son iones que disueltos en el agua de la fase acuosa del hormigón despasivan a la armadura localmente (Fig. 2) y producen un ataque en profundidad que puede originar una disminución puntual notable del diámetro de la armadura.

Por tanto, a nivel mundial se está en una fase de transición en cuanto al uso masivo del hormigón armado. Algunos países han empezado a tomar medidas de muchos tipos para dotarlo de la adecuada durabilidad. En nuestro país no existía esa conciencia colectiva hasta la evidencia de la falta de durabilidad en los hormigones de aluminoso. No hay mal que por bien no venga siempre

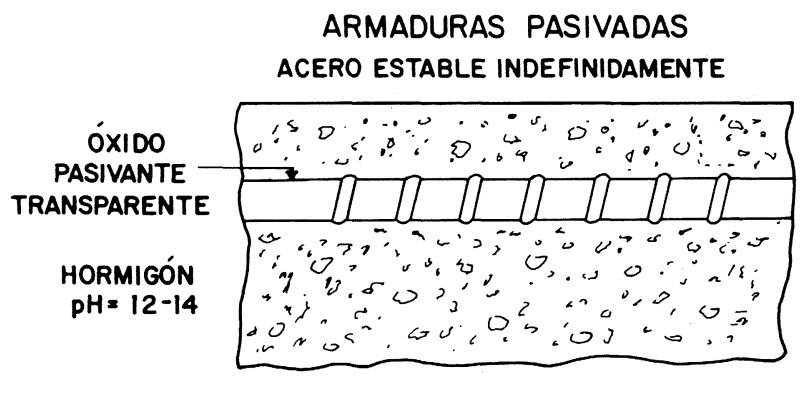

Fig. 1.-Las armaduras se conservan indefinidamente sin corrosión en un hormigón alcalino.

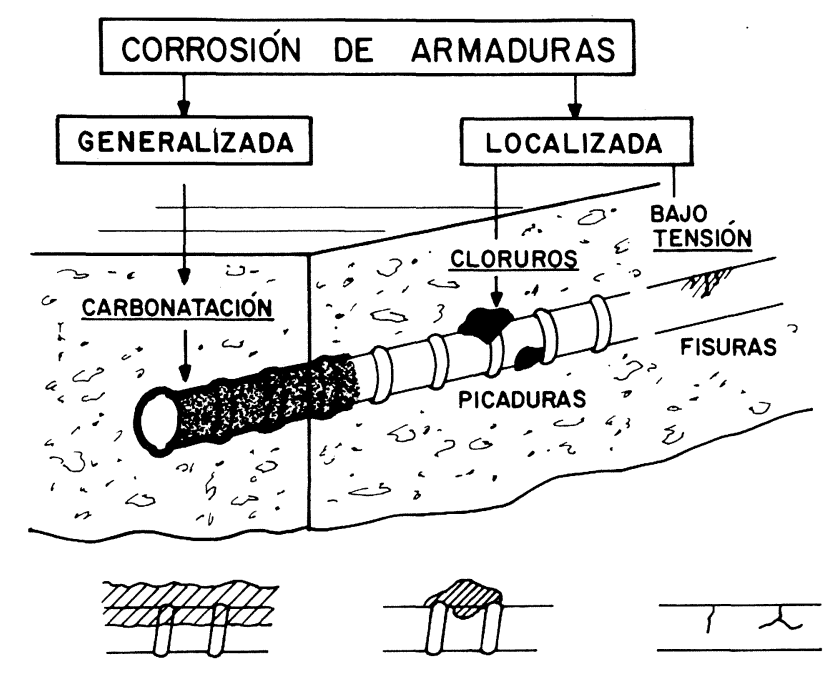

Fig. 2.-Tipos de corrosión que se pueden producir en el hormigón.

que las actuaciones que se han empezado a acometer continúen y, fuera ya de dramatismos o demagogias, se vaya construyendo un cuerpo de normativa apropiado a las necesidades aparecidas.

\section{CEMENTO ALUMINOSO}

La fase acuosa del cemento aluminoso, aunque es también alcalina $(\mathrm{pH}$ alrededor de 12,5$)$ debido a los propios álcalis que posee este cemento [14], no tiene en cambio reserva alcalina cálcica y, por tanto, es más sensible a una neutralización con sustancias ácidas o a un ataque por cloruros (la relación $\mathrm{Cl}^{-} / \mathrm{OH}^{-}$límite para despasivar es más favorable ya que al ser menor el contenido de $\mathrm{OH}^{-}$se necesitan menos cloruros para superar el límite de riesgo).

http://informesdelaconstruccion.revistas.csic.es 

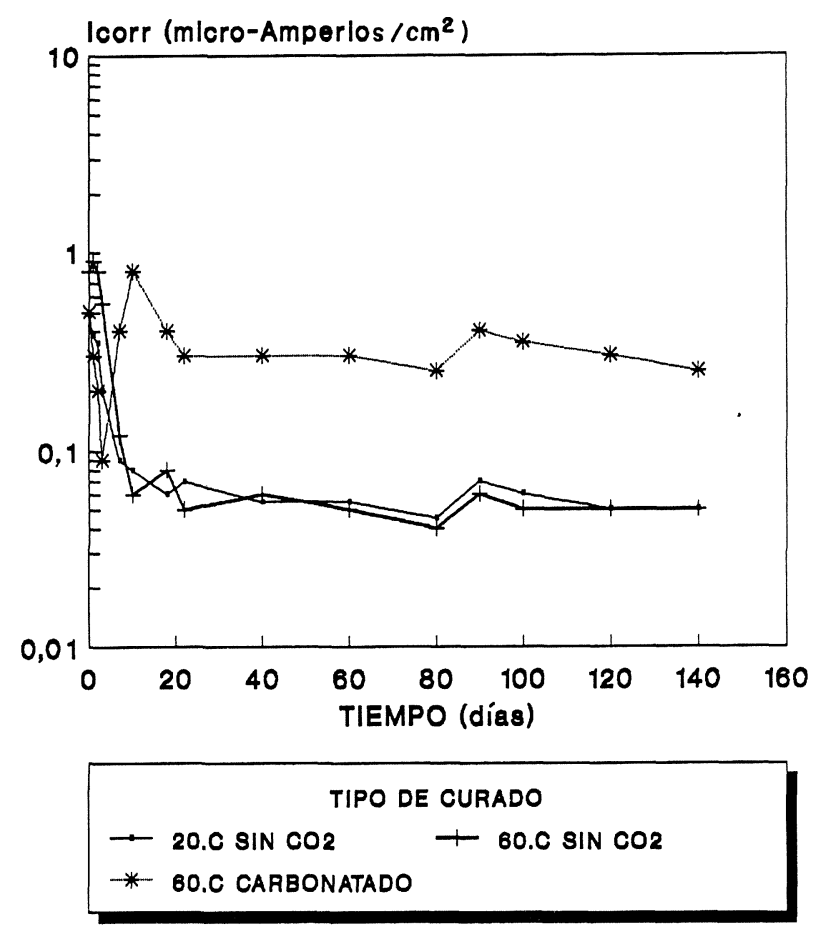

Fig. 3.-Velocidad de corrosión en el tiempo de aceros embebidos en mortero de cemento aluminoso sin transformar $(\cdot)$, transformado a fase cúbica (*) y carbonatado (+).

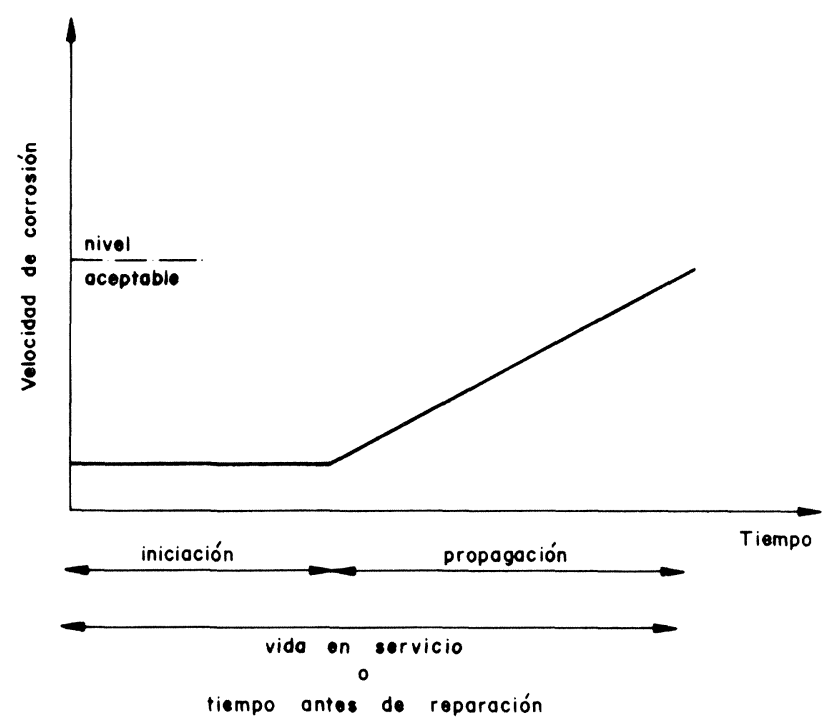

Fig. 4.-Modelo de Tuutti.

Así pues, el pH de la fase acuosa, contenida en los poros del hormigón de cemento aluminoso, es suficientemente alcalino para asegurar una pasividad estable de las armaduras mientras las condiciones de servicio no cambien.
En la figura 3 se muestran los resultados de valores de intensidad de corrosión, Icorr [15] en la que se dan los valores de redondos lisos de acero dulce embebidos en probetas de mortero fabricado con cemento aluminoso de relación $\mathrm{a} / \mathrm{c}=0,5 \mathrm{y}$ cemento/arena $=1 / 3$.

En la figura se puede apreciar que, en el caso de un curado a $20^{\circ} \mathrm{C}$ (el aluminoso se mantiene en forma de hidratos hexagonales) y posterior inmersión parcial de la probeta, no se apreció corrosión significativa durante tres años (Icorr $<0,1 \mu \mathrm{A} / \mathrm{cm}^{2}$ ).

Cuando el curado se realiza a $60^{\circ} \mathrm{C}$ durante $3 \mathrm{~h}$, se consigue que el cemento aluminoso cristalice en hidratos cúbicos. En la misma figura se aprecia también que en estas condiciones los redondos se siguen manteniendo pasivos (Icorr $<0,1 \mu \mathrm{A} / \mathrm{cm}^{2}$ ). En cambio cuando el cemento se carbonata, sí se aprecian valores de Icorr más elevados (alrededor de $0,3 \mu \mathrm{A} / \mathrm{cm}^{2}$ en las condiciones del ensayo) como muestra la figura.

Por lo cual parece que la conocida "conversión" del cemento aluminoso, es decir, el paso de las fases hexagonales a las cúbicas no supone un aumento de la velocidad de corrosión, como fue confirmado por Goñi [16] recientemente, y por lo tanto el $\mathrm{pH}$ de la fase acuosa no se altera. Sólo cuando se carbonata el hormigón, y cuando se produce una bajada importante del $\mathrm{pH}$ de la fase acuosa, es cuando se desarrolla una velocidad de corrosión significativa.

\section{VIDA ÚTIL DE ESTRUCTURAS DE HORMIGÓN}

Ésta se puede definir como el período de tiempo durante el cual la estructura conserva todas las características de funcionalidad, seguridad, resistencia y aspecto externo, con un nivel de seguridad suficiente [17].

Este concepto fue introducido en los Códigos de Cálculo de Hormigón [17] [18] en relación a posibles daños de tipo mecánico y, en los últimos años, se aplica para enmarcar la necesidad de un cálculo basado también en criterios de durabilidad.

En relación a un posible ataque por corrosión de armaduras, es ya bien introducido un esquema muy simple que se reproduce en la figura 4 y que se debe a Tuutti [19]. Este investigador divide la vida útil de una estruchttp://informesdelaconstruccion.revistas.csic.es 
tura en dos períodos: $t_{o} o$ período de iniciación y $t_{p} o$ período de propagación.

\section{Período de iniciación}

El período de iniciación $\mathrm{t}_{\mathrm{o}}$ comprende desde la fabricación de la estructura hasta el momento en que el agresivo llega hasta la armadura y la despasiva. Es decir, es el tiempo que tarde la carbonatación o los cloruros en alcanzar la armadura y empezar a corroerla. Durante este período no hay pérdida ninguna de la integridad estructural.

En cuanto a la velocidad de avance de la carbonatación, ésta depende fundamentalmente de los siguientes factores:

- proporción de $\mathrm{CO}_{2}$ en la atmósfera (alrededor del $0,03 \%$ en atmósferas rurales);

- humedad relativa de la atmósfera;

- tipo de cemento y cantidad del mismo en el hormigón;

- relación a/c.

En la actualidad está bastante verificado que la velocidad de carbonatación sigue en general una ley de tipo logarítmico que, simplificando, puede escribirse:

$$
X=k \sqrt{t}
$$

$\mathrm{X}=$ espesor de recubrimiento $(\mathrm{mm})$

$\mathrm{k}=$ constante que depende de los factores antes mencionados $\left(\mathrm{mm} / \mathrm{año}^{0,5}\right)$

$\mathrm{t}=$ tiempo (años)

Los valores de $\mathrm{k}$ que se han detectado en estructuras reales en cemento portland varían entre 2 y 15 $\mathrm{mm} / \mathrm{año}^{0,5}$, siendo su dependencia de la calidad del hormigón la siguiente:

$2<\mathrm{k}<6$........... hormigones de elevada compacidad y contenido en cemento $\left(>350 \mathrm{~kg} / \mathrm{m}^{3}\right)$

$6<\mathrm{k}<9$ hormigones de compacidad media

$\mathrm{k}>9$ hormigones porosos de baja calidad $\left(<250 \mathrm{~kg} / \mathrm{m}^{3}\right.$ y elevada relación $\mathrm{a} / \mathrm{c}$ )
Un cálculo sencillo a partir de esta fórmula lleva a deducir que, para que el frente carbonatado no llegue en 50 años hasta $20 \mathrm{~mm}$ de profundidad (recubrimiento habitual en muchas estructuras), la $\mathrm{k}$ del hormigón debería ser:

$$
\mathrm{k}=\frac{20}{\sqrt{50}}=2,82
$$

Es decir, sólo hormigones de muy elevada compacidad podrán mantener exenta de corrosión la armadura en un período de vida superior a 50 años. Todo ello, por supuesto, en hormigones situados en la atmósfera y protegidos de la lluvia, ya que en hormigones húmedos la carbonatación no penetra (en los períodos de lluvia el avance del frente carbonatado se detiene).

De la misma forma, si tenemos un hormigón con una $\mathrm{k}=7$, por ejemplo, la profundidad de carbonatación a los 50 años sería de:

$$
X=7 \times 7,07 \cong 50 \mathrm{~mm}
$$

Con la penetración de iones cloruro se podrían hacer deducciones parecidas, si bien como ya se ha comentado no se van a realizar aquí, ya que son de menor relevancia con respecto al cemento aluminoso.

Todo lo anteriormente expuesto relativo al período de iniciación se aplica a hormigones fabricados con cemento portland, ya que no se han encontrado datos similares para el cemento aluminoso. Desde luego se han estudiado bastante los mecanismos de carbonatación de este cemento, pero es curioso comprobar que no se encuentran referencias sobre a qué velocidad se carbonata o avanza hacia el interior un frente carbonatado o, ni siquiera, si este frente se produce, ya que no se conoce si la carbonatación progresa desde el exterior hacia el interior o se produce en toda la masa a la vez a partir de los poros más grandes.

Tampoco se conocen con profundidad los cambios que ocurren en la fase acuosa de los hormigones con cemento aluminoso durante la carbonatación y que, finalmente, provocan la corrosión de las armaduras. Por lo tanto no es posible dar valores de $\mathrm{k}$ característicos de estructuras fabricadas con cemento aluminoso. 


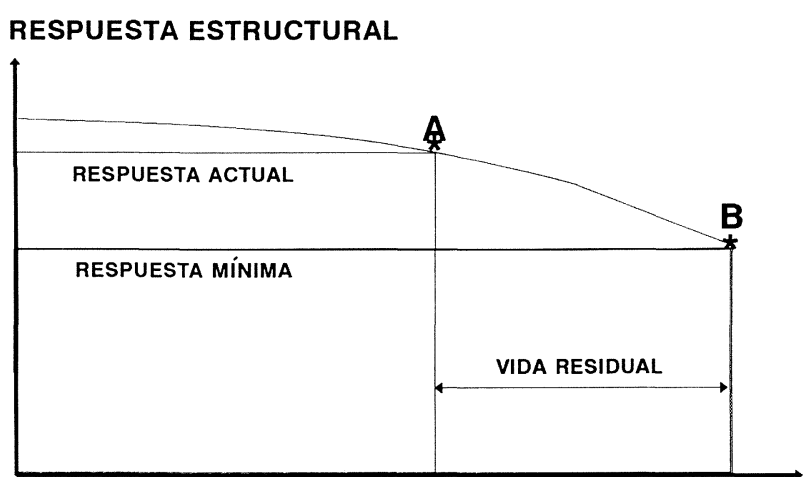

Fig. 5.-Esquema de disminución teórica de la capacidad portante en función del tiempo.

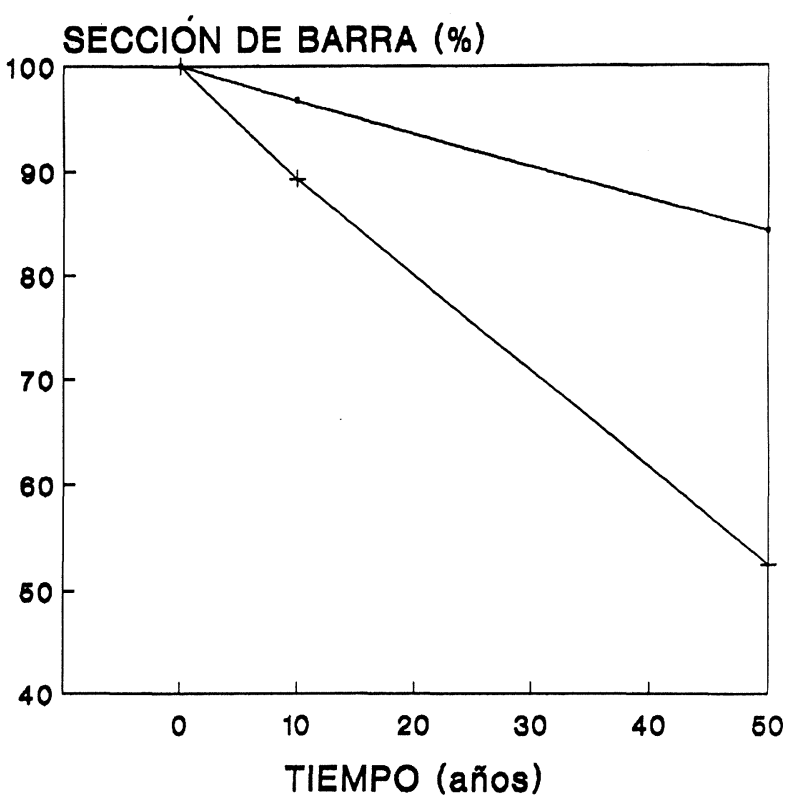

VELOC.PERDIDA SECC.

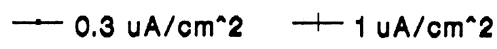

Fig. 6.-Disminución con el tiempo de la sección de un alambre de $4 \mathrm{~mm}$ de diámetro en función de la velocidad de corrosión: $0,3 \mu \mathrm{A} / \mathrm{cm}^{2}(\cdot)$ y $1 \mu \mathrm{A} / \mathrm{cm}^{2}(+)$ en el supuesto de corrosión homogénea y generalizada.

Período de propagación

Como período de propagación, $\mathrm{t}_{\mathrm{p}}$, según Tuutti, es aquel que discurre desde que la armadura se despasiva hasta que se llega a un determinado límite (que este investigador no define cuantitativamente, sino que simplemente lo define como "inaceptable").

Mientras que con respecto al período de iniciación hay un acuerdo generalizado y sólo se discrepa en las fór- mulas más idóneas para calcular la velocidad de penetración del agresivo, en cuanto al período de propagación la discusión está abierta y es controvertida. El motivo fundamental se basa en que durante esta fase de propagación sí hay ya una pérdida de la integridad estructural.

Por ello, hay autores que prefieren no considerar la posibilidad de una corrosión durante el período de vida útil teórica, lo cual resulta muy seguro pero llevaría a la necesidad de recubrimientos enormes en determinados ambientes. $Y$ entre aquellos que aceptan un cierto grado de corrosión, como no peligroso para la estructura, no hay acuerdo todavía sobre cuál es el "límite inaceptable" a partir del cual la estructura deja de ser segura, o pierde funcionalidad o simplemente estética.

No se va a entrar aquí en detalle sobre los distintos aspectos de este debate, que pueden ser encontrados por los lectores interesados en la bibliografía especializada [20] [21] [22], pero sí se van a apuntar los más relevantes.

Para ello en la figura 5 se ha representado esquemáticamente la pérdida paulatina de integridad estructural que se puede producir por una corrosión progresiva de las armaduras. En realidad esta corrosión afecta a tres características del hormigón armado:

- Características mecánicas del acero al disminuir su sección por la corrosión (puede haber también una pérdida de ductilidad no bien verificada todavía).

- Características mecánicas del hormigón que rodea a la armadura, debido a la fisuración del recubrimiento por efecto de la expansión de los óxidos que se generan en la corrosión.

- Pérdida de adherencia entre el hormigón y el acero como consecuencia de los dos fenómenos anteriores.

En realidad estos tres fenómenos dependen de la velocidad a la que se produzca la corrosión, que a su vez es función principalmente del contenido en humedad del hormigón.

En la figura 6 se ha representado la pérdida teórica de la sección de una barra de $4 \mathrm{~mm}$ de diámetro en función de dos velocidades de corrosión $\left(0,3 \mu \mathrm{A} / \mathrm{cm}^{2}\right.$ y $1 \mu \mathrm{A} / \mathrm{cm}^{2}$ ) y suponiendo una corrosión homogénea. Ambas son habituales en hormigones carbonatados y húmedos de cemento aluminoso. 
Se puede comprobar que con la velocidad más elevada de las dos, en 30 años se puede ya haber perdido un $40 \%$ de la sección de las barras. Muy probablemente para entonces ya la adherencia puede haberse afectado sensiblemente o, si el recubrimiento era muy pequeño, éste puede haberse fisurado o desprendido.

A la vista de esta situación resulta difícil definir cuál es el "límite inaceptable", antes aludido, donde situar el fin de la vida útil considerada en el proyecto. Las propuestas actualmente en estudio por un grupo de trabajo del CEN [23] [24], se considera este límite como una pérdida del $5 \%$ de la sección de la barra, o bien la aparición de fisuras en el recubrimiento no superiores a $0,3 \mathrm{~mm}$ de ancho, siempre considerando elementos armados, no pretensados.

En el caso de hormigones pretensados la situación cambia radicalmente, y aquí no se debería aceptar ninguna corrosión dentro del período de vida útil. Es decir, hay que considerar acabada la vida de una estructura pretensada cuando el agresivo llega a la armadura.

Un importante aspecto a comentar -aunque no se hayan abordado aquí los aspectos específicos de las transformaciones que sufre el cemento aluminoso durante su envejecimiento, pero que hay que tener en cuenta a la hora de evaluar la vida residual de una estructura fabricada con este cemento- es la posible pérdida de resistencia mecánica que puede llevar aparejada todo el proceso de degradación de las fases del cemento aluminoso. Así, mientras en el caso del cemento portland el período de iniciación no suponía una pérdida de la integridad estructural, en el caso del aluminoso esta pérdida puede aparecer y hay que tenerla en cuenta en la evaluación que se efectúe. En este caso el diagrama de disminución de capacidad portante de la estructura seguirá de forma esquemática la tendencia mostrada en la línea $\mathrm{A}$ de la figura 7 .

Finalmente se puede comentar que el fin de la vida útil de una estructura no tiene porqué coincidir con el momento de una reparación. Esta última tiene que estar basada en criterios económicos y sociales además de los puramente técnicos y puede resultar más adecuado retrasar la reparación, o incluso no hacerlo, y simplemente controlar la evolución de la estructura. Es por ello por lo que el fin teórico de la vida útil debe situarse suficientemente lejos de cualquier riesgo de daño estructural significativo.
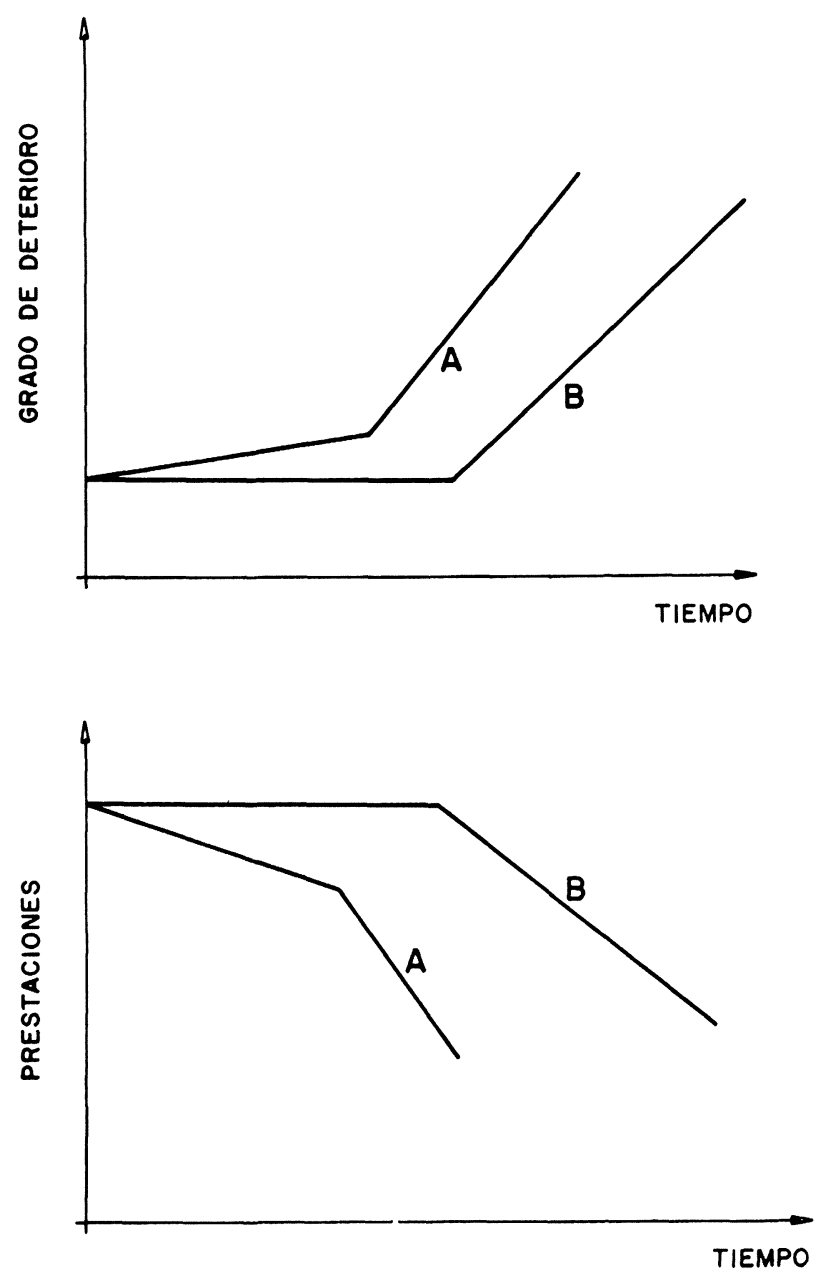

Fig. 7.-Esquema de aumento del deterioro o disminución de prestaciones en el caso: A) que se produzca pérdida de resistencias mecánicas del hormigón o B) que sólo se deteriore la armadura.

\section{INSPECCIÓN DE CORROSIÓN EN ESTRUCTURAS DE CEMENTO ALUMINOSO}

Ya se ha mencionado que en el presente artículo no se abordan aspectos relativos al propio hormigón, es decir, no se aludirá en este apartado a los métodos para identificar la presencia o no de cemento aluminoso, sino aquellos relativos a valorar la posible corrosión y su grado de avance, así como en lo posible indicar su evolución futura.

Para evaluar la corrosión, lo más simple aparentemente es realizar una observación visual detallada. Hay que evaluar si aparecen grietas o manchas de óxido y siempre que sea posible realizar alguna pequeña perforación y observar directamente la armadura. 
Las diversas situaciones que pueden aparecer son:

a) Si no hay señales externas de corrosión y las armaduras están limpias, y el hormigón está completamente transformado, hay que suponer que no se producirán corrosiones en el futuro a no ser que aparezcan humedades inesperadas. Es decir, las armaduras pueden conservarse exentas de óxido en hormigón de aluminoso completamente carbonatado (se han encontrado diversos casos reales). Pues bien, el supuesto de evolución futura es que la situación se mantendrá igual a no ser que cambien las condiciones de servicio (especialmente que aumente la humedad).

b) En cambio si las armaduras están limpias en el momento de hacer la inspección pero el aluminoso está a medio transformar, entonces sí que hay que trabajar con la hipótesis de una posible corrosión futura, a no ser que el ambiente sea siempre seco (HR medias menores del 60\%).

c) Lo opuesto es que se detecte la presencia de fisuras en el recubrimiento o manchas de óxido. Entonces se recomienda también acceder a observar directamente la armadura y la posible presencia de óxidos. Si el recubrimiento no se ha desprendido, y sólo presenta fisuras en general, la corrosión no será importante (a no ser que la relación diámetro barra/espesor de recubrimiento sea muy pequeño). En cambio si hay zonas del recubrimiento ya descascarilladas, es de esperar corrosiones más intensas. Resulta aquí de vital importancia fijarse en si la corrosión es general o más localizada, con el fin de prever un posible fallo local.

Si la corrosión está presente, el factor fundamental para que se pueda mantener es la presencia de elevadas humedades ambientales o agua (bajantes, desagües, filtraciones, etc). Si el elemento no se va a sustituir es fundamental cortar radicalmente el acceso de humedad a la armadura, lo que por otro lado resulta muy complejo ya que el hormigón actúa de esponja que no se seca fácilmente y es difícil de impermeabilizar por las posibles presiones de vapor que genera internamente durante los cambios de temperatura.

Además de la simple observación visual, ya es posible en nuestro país la medida visual directa en obra de la velocidad de corrosión [25], si bien esta medida sólo es interpretable por especialistas, ya que exige una experiencia previa para su correcto uso. Consiste la técnica (c) Consejo Superior de Investigaciones Científicas Licencia Creative Commons 3.0 España (by-nc) en una medida de la llamada "Resistencia de Polarización", valor a partir del cual es posible calcular los Icorr que presenta la armadura. Con estos valores, y siguiendo el esquema que se mostró en la figura 6 , se puede hacer una cierta predicción de la evolución futura de la pérdida de sección de la barra. Esta predicción es todavía muy inexacta, pero al menos mucho más aproximada que la que se puede realizar con una simple observación visual.

Valores de Icorr han sido medidos en el patrimonio edificado perteneciente a la Empresa Pública ADIGSA de la Consejería de Bienestar Social de la Generalitat de Cataluña, si bien los resultados no pueden ser publicados todavía. Como avance puede decirse que los valores mayores detectados allí no sobrepasan los $2 \mu \mathrm{A} / \mathrm{cm}^{2}$ habitualmente.

La traducción de estas medidas de velocidad de corrosión en pérdida de capacidad portante del elemento estructural es todavía arriesgada, aunque más vale hacer esta extrapolación en base a los datos cuantitativos de Icorr complementados con las observaciones de:

- detalle de armado;

- geometría del elemento;

- estado tensional de los aceros;

- presencia de defectos como grietas, fisuras, manchas, etc.;

- resistencia mecánica residual del hormigón y grado de transformación del aluminoso,

que hacerlas en base a la posible experiencia o intuición del observador.

En cualquier caso los cálculos relativos al comportamiento estructural futuro son imprescindibles si hay lesiones, con el fin de decidir la conveniencia o no de sustituir el elemento o simplemente repararlo.

De forma esquemática se puede resumir el camino a seguir en una inspección de las armaduras:

1) Identificar si hay lesiones visibles y romper el recubrimiento en alguna pequeña zona para acceder a ver la armadura directamente:

* ¿Hay óxido visible?

- Si es que NO, hay que ampliar los lugares de muestreo para comprobar que no hay óxido en más de http://informesdelaconstruccion.revistas.csic.es 
un sitio y estar seguros de que no es una casualidad. Si la armadura está limpia y es aluminoso, hay que pensar en volver a hacer una inspección visual al cabo de varios años (5 a 10 en función de la humedad ambiental).

- Si es que SÍ hay que graduar la importancia de la corrosión (ver 2).

2) Graduar la importancia de la corrosión:

* Para ello se pueden usar datos de velocidad de corrosión o hacerlo en función de la cantidad de óxido visible:

- LEVE: óxido superficial y no hay pérdida de sección.

- MODERADA: óxido más abundante con ligera pérdida de sección.

- GRAVE: pérdida de sección notable, óxido abundante, o grietas y fisuras.

3) A partir de estos datos se decide la frecuencia de inspección futura o la urgencia de intervención. Así habrá que intervenir inmediatamente (sea reparar, evitar escapes de agua o sustituir el elemento):

- siempre que la corrosión sea grave;

- cuando la corrosión sea moderada y el elemento es pretensado;

- cuando la corrosión sea moderada, el elemento es armado pero a través de un recálculo su resistencia residual es deficiente.

En los demás casos (corrosión leve o moderada con suficiente resistencia residual) se realizarán medidas correctoras e inspecciones futuras cuya frecuencia no será inferior a un año y dependerá de las circunstancias en las que se encuentre el elemento en la estructura y su geometría.

Se ha de intentar preservar el hormigón siempre lo más seco posible.

Todas estas indicaciones son orientativas y tratan simplemente de ayudar, si bien el técnico que efectúe la inspección ha de tratar de documentarse en esta especialidad con manuales o libros escritos por especialistas y, finalmente, utilizar el sentido común y su buen saber que, sin duda, le llevará a consultar con especialistas en caso de duda.

Para finalizar, en cuanto a reparación de los elementos de cemento aluminoso también es un tema delicado, ya que si el aluminoso está envejecido no parece conveniente ni ponerlo en contacto con aluminoso nuevo ni con cementos en base portland. Tampoco se recomienda acudir a morteros de resina epoxi, que si bien pueden tener una buena apariencia al principio, no hay acuerdo sobre su capacidad de protección hacia la armadura a largo plazo. Por tanto, no queda más que acudir a morteros preparados y comercializados por firmas de solvencia reconocida que cumplan las siguientes condiciones: a) sean bajos en álcalis; b) contengan resinas o humo de sílice que puedan disminuir algo su alcalinidad (su neutralización completa dejaría desprotegidas las armaduras); c) que sean bajos en sulfatos (no estén basados en sulfoaluminatos); d) que tengan retracción compensada y una buena adherencia al hormigón viejo con el fin de no dejar fisuras en las juntas de unión. Por último, como siempre, evitar el agua, las condensaciones o las humedades elevadas.

\section{BIBLIOGRAFÍA}

[1] P. STIGLITZ - Silicates Industriels, Ab (1972) 93-99.

[2] C.M. GEORGE - Revue des Matériaux des Construction 701, Abril (1976) 201.

[3] A.M. NEVILLE - High Alumina Cement Concrete - 1975 The Construction Press - Lancaster (U.K.).
[4] C. ANDRADE, S. GOÑI, J.L. SAGRERA y C. ALONSO - Revista BIA (Col. Aparejadores de Madrid) n. 143 - Marzo (1991) 83-97.

[5] ITEC - Recomendaciones para el reconocimiento sistemático y la diagnósis rápida de viguetas construídas con cemento aluminoso - Generalitat de Cataluña (1991). 
[6] G. VERBECK - PCA Boletín n. 87, Feb. (1958).

[7] M. HAMADA - Proceedings 5. Congreso Internacional de la Química de cemento - Tokyo (1968) sesión III.3, (343-409).

[8] M. VENUAT, J. ALEXANDRE - CERILH Publicación n. 195 (1977).

[9] J.A. GONZÁLEZ, S. ALGABA y C. ANDRADE - British Corrosion Journal val. 15 (1991), 135.

[10] J. NAUMANN, H. BAUMEL - Archiv. für Eisenhuttenwesen n. 2 (1961) 89-94.

[11] D. REHM - Betonstein-Zeitung n. 12 (1963) 651-661.

[12] C. del OLMO - Revista Iberoamericana de Corrosión y Protección, Abril (1977) 37-42.

[13] T. VÁZQUEZ, F. TRIVIÑO, A. RUIZ DE GAUNA - Monografía n.o 334 del ICCET (1976).

[14] M.T. GAZTAÑAGA - Tesina de Licenciatura - Univ. Complutense de Madrid (1989).

[15] M. PÉREZ - Tesis Doctoral - Universidad Complutense de Madrid (1981).
[16] S. GOÑI, C. ANDRADE y C.L. PAGE - Cement and Concrete Research vol. 21 (1991) 635-646.

[17] EH-91 - Instrucción para el Proyecto y Ejecución de obras en Masa y Armado - MOPT, Madrid (1991).

[18] ENV-1992.1.1 - Eurocode 2: Design of concrete structures. Part 1: General rules and rules for buildings - CEN - Dec (1991).

[19] K. TUUTTI - Corrosion of steel in concrete - Tesis Doctoral CBI - Estocolmo (Suecia) 1982.

[20] L. MASTERS - Matériaux et Constructions - Rilem vol. 19, n. 114 (1986) 417-422.

[21] T. SNECK - 3rd Int. Conference on the Durability of Building Materials and Components - Espoo- Finland - Ag (1984) 12-40.

[22] CEB-Bolletin n. ${ }^{\circ}$ 182. Guide to Durable Concrete Structures.

[23] CEN TC 104/WG1/TG1/ Panel 1 - Documentos de trabajo.

[24] L. PARROTT et al - CEN TC 104/TG1/WG1/Panel 1: Design for avoiding damage due to carbonation - induced corrosion.

[25] S. FELIU, J.A. GONZÁLEZ, S. FELIU Jr. y C. ANDRADE - ACI Materials Journal, vol. 87, n.o 5 (1990) 457-460.

\section{publicación del ICCET/CSIC \\ INSPECCION DE OBRAS DAÑADAS POR CORROSION DE ARMADURAS}

El presente Manual va dirigido principalmente a técnicos especializados y laboratorios que tienen que intervenir en el dictamen de la situación de deterioro de estructuras de hormigón armado dañadas por corrosión de armaduras.

Comienza con un resumen recordatorio de los factores principales a los que se pueden deber los daños prematuros por corrosión de armaduras, para seguir con algunas indicaciones de cómo se deben realizar las inspecciones, y de los ensayos y la metodología que se recomienda realizar para poder dictaminar con precisión las causas de daño.

A continuación se hacen una serie de comentarios sobre la vida residual de estructuras dañadas, sobre el riesgo de corrosión futura, el seguimiento necesario de una estructura reparada y una breve enumeración de métodos de reparación y consideraciones básicas a tener en cuenta en la recomendación de un determinado método. Se aporta una breve relación bibliográfica.

Finalmente se incluyen en forma de ficha la descripción de algunos casos de corrosión de armaduras detectados en nuestro país.

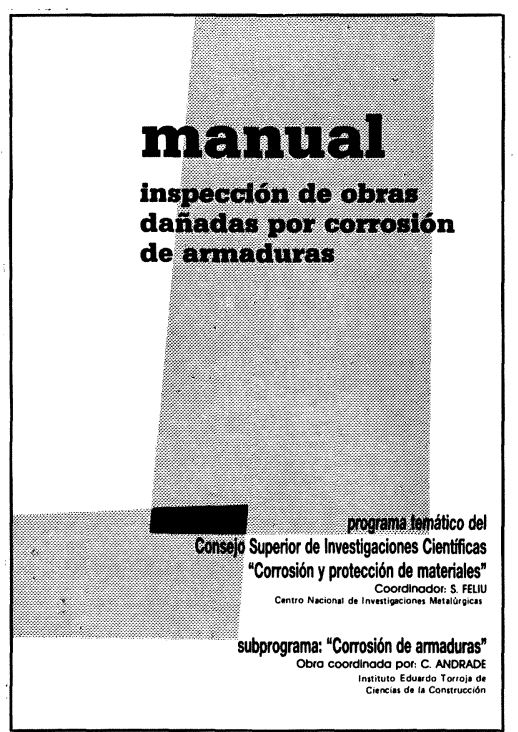

http://informesdelaconstruccion.revistas.csic.es 